\title{
TEMA-2017: Análisis retrospectivo descriptivo de tamizaje combinado en primer trimestre de embarazo en el periodo comprendido de febrero 2016 a marzo 2017, Unidad de Medicina Materno Fetal, Hospital San Juan de Dios
}
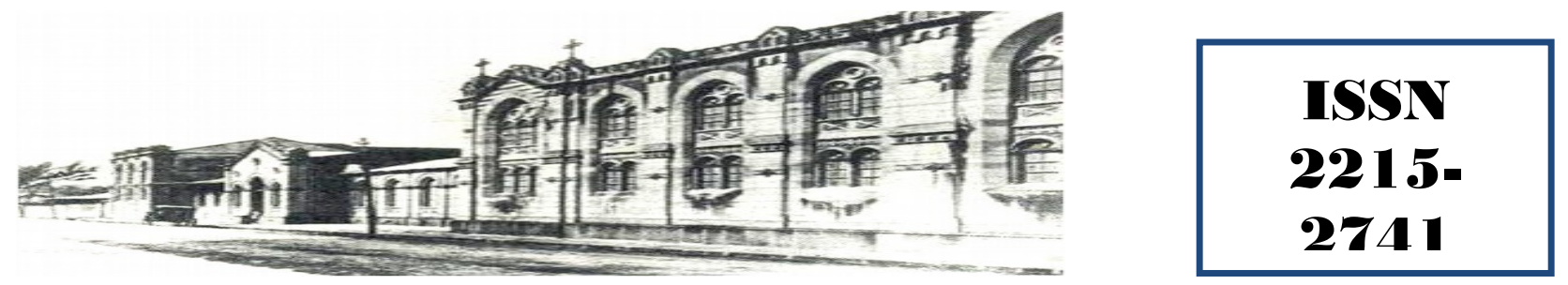

Haspital San quan de Dias. San José. Casta Rica. Fundada en 1845

Recibido:

$08 / 07 / 2017$

Aceptado:

$15 / 08 / 2017$

Carina Breuning Velásquez ${ }^{1}$

Joaquín Bustillos Villavicencio ${ }^{2}$

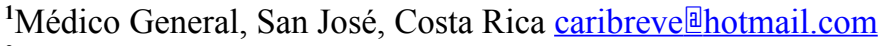

${ }^{2}$ Médico Asistente Especialista, Unidad Medicina Materno Fetal, Hospital San Juan de Dios, San José, Costa Rica, CCSS joaquin.bustillos@hotmail.com

\section{RESUMEN}

Se realiza un estudio retrospectivo descriptivo en el Hospital San Juan de Dios, San José, Costa Rica, durante el periodo de febrero 2016 a marzo 2017, con un total de 37 pacientes a las cuales se les realiza tamizaje combinado en el primer trimestre de embarazo, dentro del cual se evalúan las variables de edad materna, métodos bioquímicos y ecográficos que puedan predecir en conjunto el riesgo de alteraciones cromosómicas fetales en el embarazo.

Utilizando el tamizaje combinado como método no invasivo se pretende identificar las gestaciones de alto riesgo, realizando así un número mínimo de procedimientos invasivos para detectar el mayor número de casos.

Mediante éste tamizaje se identifican cuatro pacientes con alto riesgo de presentar aneuploidía en el embarazo.

\section{PALABRAS CLAVE}

Protocolo; tamizaje combinado; primer trimestre; aneuploidía; trisomía.

\section{ABSTRACT}

A retrospective descriptive study is conducted at the San Juan de Dios Hospital, San José, Costa Rica, during the period from February 2016 to March 2017, with a total of 37 patients from which a combined screening during the first trimester of pregnancy was conducted, evaluating maternal age, biochemical and sonographic methods that together can predict the risk of fetal chromosomal alterations during pregnancy.

The purpose of using combined screening as a non invasive method is to identify high risk ges- 
tations and to minimize the number of invasive procedures to detect the highest number of cases. Four patients with higher risk of aneuploidy during pregnancy were identified through this screening.

\section{KEY WORDS}

Protocol; combined screening; first trimester; aneuploidy; trisomy

\section{INTRODUCCIÓN}

El presente trabajo está basado en los resultados obtenidos del tamizaje combinado en el primer trimestre de embarazo que sugieran aneuploidía fetal, enfocados en las trisomías 21, 13 y 18, síndrome de Down, Patau y Edwards respectivamente; con el fin de educar a los padres y prever las causas de morbilidad y mortalidad perinatal asociadas.

Se establece el protocolo de ecografía del primer trimestre y tamizaje de alteraciones cromosómicas y estructurales en el primer trimestre del embarazo para el Servicio de Obstetricia del Hospital San Juan de Dios y éste se realiza durante el periodo comprendido desde febrero 2016 a marzo 2017. El objetivo del tamizaje pretende reducir la cantidad de procedimientos invasivos durante el embarazo que pueden llevar a riesgo de pérdida de éste, además de reducir los costos que esta medida genera. ${ }^{(1)}$

Debido a que el tamizaje basado en la edad materna representa un método de poca sensibilidad y especificidad se utiliza el método combinado que incluye las variables de edad materna, métodos bioquímicos y ecográficos que juntos proporcionan una precisión muy superior, alcanzando una tasa de detección para trisomía 21 de un $90 \%$ y un $5 \%$ de falsos positivos. ${ }^{(1,2)}$

Los criterios para indicar el estudio son:

- Menores de 15 años y mayores de 35 años de edad.

- Antecedente de aneuploidía así como malformación o síndrome genético.

- Diabéticas pregestacionales.

- Epilépticas

- Antecedente de 3 o más abortos

- Pacientes con embarazo producto de fertilización in vitro
- Pacientes con exposición a radiación en el primer trimestre.

El protocolo contempla dos tipos de exploración: el ultrasonido entre las semanas $11-13^{+6}$ de gestación y análisis bioquímicos en sangre materna de la fracción beta de la gonadotropina coriónica humana $(\beta-h C G)$ y la proteína específica-A asociada al embarazo (PAPP-A). Ambos resultados se expresan en múltiplos de la mediana (MoM) para edad gestacional. En trisomía 21 los resultados de $\beta$-hCG se encuentran elevados y PAPP-A reducidos, mientras que en el caso de trisomía 13 y 18 ambos se encuentran reducidos.

Los marcadores ecográficos de aneuploidía más importantes son la translucencia nucal (TN) y el hueso nasal. Un aumento de la translucencia nucal traduce acumulación de líquido detrás del cuello fetal el cual se observa en la evaluación ultrasonográfica del primer trimestre y se asocia con trisomía 21 , en un $75 \%$ de los fetos con T21 se reporta TN aumentada. La ausencia de hueso nasal no necesariamente aumenta la tasa de detección de síndrome de Down, sin embargo puede ser usado como marcador para disminuir los falsos positivos.

Otros marcadores de la valoración ecográfica del primer trimestre son el ductus venoso y la regurgitación tricúspidea, ambos con mayor incidencia en fetos con trisomía 21 comparados con fetos euploides, y así aumentan la detección de aneuploidía y reducen las tasas de falsos positivos sin embargo no son tomados en cuenta en el tamizaje combinado ya que su presencia aislada no es indicador de aneuploidía o patología perinatal y su estudio amerita ser realizado por personal altamente entrenado y se reserva para población con riesgo intermedio-alto. ${ }^{(1,2,9)}$

Dentro del protocolo también se contempla la posibilidad de realizar la evaluación bioquímica durante el segundo trimestre a casos que no hayan sido tamizados durante el primer trimestre. En este caso se recomienda por parte de ACOG (Congreso Americano de Ginecología y Obstetricia) la evaluación cuádruple de marcadores séricos maternos: alfa feto proteína, $\beta \mathrm{HCG}$, estriol e inhibina A, así como la evaluación ecográfica entre la semanas 18 y 20 de gestación. Los marcadores de ultrasonido en el segundo trimestre incluyen: quiste del plexo coroideo, foco ecogénico intracardiaco, aumento de pliegue nu- 
cal, vejiga ecogénica, arteria umbilical única, ventrículomegalia, ausencia o hipoplasia del hueso nasal, arteria subclavia derecha aberrante, hidronefrosis y acortamiento del femur y/o del húmero. Estos marcadores se presentan con una incidencia aumentada en fetos con aneuploidía comparada a fetos euploides. ${ }^{(1,2,9,12)}$

El programa informático utilizado para el tamizaje combinado realiza un cálculo básico donde integra las tres variables fundamentales y la edad gestacional: edad, translucencia nucal y resultado del análisis bioquímico y lo expresa en términos de fracción. Se utiliza como punto de corte para screening positivo el riesgo de aneuploidía a los 35 años $1 / 270$. $^{(1)}$

En caso de screening positivo se recomienda el estudio de cariotipo fetal, el método de elección es biopsia de vellosidades coriónicas preferiblemente entre las semanas $11-13^{+6}$. Después de la semana 15 de gestación el método de elección para el estudio es la amniocentesis.

La biopsia de vellosidades coriónicas se debe realizar después de las 10 semanas de gestación ya sea por vía transabdominal o transcervical dependiendo de la experiencia y preferencia del operador o localización de la placenta. Las complicaciones relacionadas a este procedimiento incluyen el riesgo de pérdida del embarazo que varía en los reportes de un 0.2 a $2 \%$ y el sangrado vaginal que se ha reportado en un $10 \%$ de los casos reportados. ${ }^{(1,11,13)}$

Factores asociados con riesgo aumentado de perdida fetal posterior a biopsia de corion descritos en estudios retrospectivos previos fueron raza materna africana-americana, al menos dos aspiraciones/inserciones de aguja, sangrado abundante durante el procedimiento, edad materna menor a 25 años y edad gestacional menor a 10 semanas.

Niveles bajos de PAPP-A están relacionados con problemas placentarios-preeclampsia por lo que se sugiere que también estén involucrados en un mayor riesgo de pérdida. ${ }^{(13)}$

La amniocentesis debe ser realizada posterior a las $15^{+0}$ semanas de gestación por vía transabdominal guiada por ultrasonido. Las complicaciones reportadas posteriores al procedimiento incluyen el riesgo de pérdida fetal que varía de 0.1 a $1 \%$, aumento de riesgo de salida de líquido am- niótico hasta las 24 semanas de gestación. Se reporta bajo riesgo de corioamnioitis e infección uterina posterior a amniocentesis, riesgo inferior a $0.7 \%$.

Por si solos la presencia de translucencia nucal aumentada y anomalías estructurales fetales están asociados a un mayor riesgo de pérdida fetal, este riesgo se aumenta seguido de procedimientos invasivos como la biopsia de vellosidades coriónicas y amniocentesis.

Otro estudio practicado en Estados Unidos es la muestra de sangre fetal, la cual se realiza por vía transabdominal después de las $18^{+0}$ semanas, para investigar principalmente mosaicismo cromosómico.

Criterios de eligibilidad para el procedimiento invasivo diagnostico:

- Alto riesgo para alteraciones cromosómicas fetales.

- Alto riesgo de enfermedades genéticas o metabólicas.

- Alto riesgo de infecciones perinatales.

Previo a cualquier procedimiento invasivo se debe brindar a la familia consejería detallada y concientizar sobre los riesgos asociados. ${ }^{(11,13)}$

\section{DISCUSIÓN}

El análisis de los resultados de tamizaje combinado en el primer trimestre de embarazo, realizado en el periodo de un año en el Hospital San Juan de Dios refleja un total de cuatro pacientes en alto riesgo detectadas por este medio. En sujetos de riesgo la edad materna promedio fue de 36 años, lo cual concuerda con la estadística universal que pone como factor de riesgo principal la edad materna avanzada correlacionada con la presencia de aneuploidía fetal.

Dentro de estos resultados cabe resaltar el de la translucencia nucal de un feto que se reporta en $3,9 \mathrm{~mm}$, superior al corte asociado con trisomía 21. Sin embargo al dar seguimiento a los casos identificados de alto riesgo, después de una adecuada consejería a las pacientes y sus familiares se reporta únicamente una paciente la cual está pendiente de realizarse la amniocentesis. Dos de las pacientes restantes rechazaron el procedimiento y una perdió el control en el Servicio. Por 
lo tanto contamos con un sesgo de información en este caso.

Este estudio concluyó un $10 \%$ de casos afectados $\mathrm{y}$ al igual que lo sugerido por fuentes extranjeras, en nuestro medio se le realizará amniocentesis a la paciente que aceptó el procedimiento, que co-

Debido a que nos encontramos en un sistema de seguridad social, en un país con recursos económicos limitados, se realiza el tamizaje seleccionado para pacientes con ciertos criterios de inclusión que pueden implicar mayor riesgo para la gestación, por lo tanto no se cuenta con un volumen de pacientes significativo.

Actualmente en otros países, se desarrolla una modalidad basada en el análisis de ADN de células libres en sangre materna. Este método cuenta con la habilidad de detectar pequeños aumentos en la cantidad de un cromosoma respectivo en plasma materno en un embarazo con trisomía. Estudios demuestran que éste medio puede detectar hasta un $99 \%$ de casos de trisomía $21,97 \%$ de trisomía 18 y $92 \%$ de casos de trisomía 13 . La tasa de falsos positivos es de $0.1 \%$ para T21, $0.2 \%$ para $\mathrm{T} 18$ y $0.2 \%$ para $\mathrm{T} 13$ respectivamente. Los hallazgos en la realización del cribado de ADN de células libres en sangre materna en población general asemejan a los de estudios previos en embarazos de alto riesgo.

Se ha demostrado que éste método es factible para tamizaje de rutina y permite un diagnóstico de aneuploidía confiable, con tasas de falsos positivos significativamente menores que las de tamizaje combinado. ${ }^{(14,15)}$

A pesar de que a nivel público no se cuenta con este estudio, lo ideal es que toda paciente embarazada por lo menos cuente con un ultrasonido del primer trimestre que incluya la medida de la translucencia nucal, la cual es un marcador no solo de alteraciones cromosómicas fetales sino también de defectos cardiacos y otros síndromes genéticos.

\section{CONCLUSIONES}

El tamizaje combinado del primer trimestre de embarazo representa una estrategia de cribado adecuada en esta población para disminuir fundamentalmente los procedimientos invasivos diag- rresponde respectivamente a un $1,85 \%$ de las pacientes tamizadas

nósticos y sus complicaciones en el caso de sospecha de aneuploidía en el feto.

En nuestro estudio, a pesar de ser una muestra de embarazadas de alto riesgo, se detectaron 4 pacientes con riesgo de cromosomopatías que equivale al $10 \%$ de la población estudiada, concordando con datos publicados y demostrándose que la asesoría al igual que el cribado es fundamental para el seguimiento final de estas pacientes.

Lo anterior confirma que el ultrasonido dirigido a tamizar pacientes con algún riesgo en el primer trimestre de la gestación, debe ser un estudio fundamental y obligatorio como medida incluso de captación inicial de la paciente durante un embarazo y, que combinado con otras variables epidemiológicas y biofísicas de esta población, nos estratifican temparanamente el riesgo individual en cada paciente y así podemos ofrecer una atención de mayor calidad en esta etapa tan determinante del ser hurmano.

\section{BIBLIOGRAFÍA}

1. Bustillos Villavicencio J. Protocolo: Ecografia de primer trimestre y screening de alteraciones cromosómicas y estructurales en el primer trimestre del embarazo. Servicio de Obstetricia, Hospital San Juan de Dios

2. Rao R., Platt L. D. Ultrasound screening: status of markers and efficacy of screening for structural abnormalities. Seminars in perinatology. WB Saunders. 2016; 67-78

3. Nicolaides KH, Heath V, Cicero S. Increased nuchal translucency at 11-14 weeks. Prenat Diagn 2002;22:308-315

4. Nicolaides KH, Sebire N, Snijders R. Nuchal translucency and chromosomal defects in: The 11-14 week scan. The diagnosis of fetal abnormalities. Diploma in fetal medicine series, Ed. KH Nicolaides. 1st Ed. London The Parthenon Publishing Group 1999 pp 3-65 
5. Cicero S, Curcio P, Papageorghiou A, Sonek J, Nicolaides KH. Absence of nasal bone in fetuses with trisomy 21 at 11-14 weeks of gestation: an observational study. Lancet 2001;358:16651667

6. Howard C. Biochemical screening for Down syndrome. Eur J Obstet Gynecol Reprod Biol 2000;92:97-101

7. Souka A, Krampl E, Bakalis S, Heath V, Nicolaides KH. Outcome of pregnancies in chromosomally normal fetuses with increased nuchal translucency in the first trimester. Ultrasound Obstet Gynecol 2001;18:9-17

8. Souka A, Snjiders R, Novakov A, Soares W, Nicolaides KH. Defects and syndromes in chromosomally normal fetuses with increased nuchal translucency thickness at 10-14 weeks of gestation. Ultrasound Obstet Gynecol 1998;11:391-400

9. Chi T, Huggon I, Zosmer N, Nicolaides $\mathrm{KH}$. Incidence of major structural cardiac defects associated with increased nuchal translucency but normal karyotype. Ultrasound Obstet Gynecol 2001;18:610-614

10. Nicolaides KH. Screening for chromosomal defects. Ultrasound Obstet Gynecol 2003:21;313-321

11. Alfirevic Z, Sundberg K, Brigham Amniocentesis and chorionic villus sampling for prenatal diagnosis. Cochrane Database Syst Rev. 2003;(3):CD003252

12. Agathokleous, M., Chaveeva, P., Poon, L. C. Y., Kosinski, P., \& Nicolaides, K. H. Meta-analysis of second-trimester markers for trisomy 21. Ultrasound in Obstetrics \& Gynecology. 2013; 41: 247-261

13. GHI, T., et al. ISUOG Practice Guidelines: invasive procedures for prenatal diagnosis. Ultrasound in Obstetrics \& Gynecology. 2016; 48: 256-268

14. Quezada M.S, Gil M.M, Francisco C., Oròsz G. \& Nicolaides K.H. Screening for trisomies 21, 18 and 13 by cell-free DNA analysis of maternal blood at 1011 weeks gestation and the combined test at 11-13 weeks. Ultrasound Obstet Gynecol. 2015; 45: 36-41.

15. Gil M.M., Quezada M.S., Revello R., Akolekar R., Nicolaides K.H. Analysis of cell-free DNA in maternal blood in screening for aneuploidies: updated meta-analysis. Ultrasound Obstet Gynecol 2015; 45: 249-266.

\section{CONFLICTO DE INTERÉS Y/O AGRADECIMIENTOS}

Los autores declaran que no existió ningún conflicto de interés en el presente reporte. 
Tabla 1: Sujetos de riesgo en análisis de tamizaje combinado.

Fuente: Perinatología HSJD

\begin{tabular}{|lcccc|}
\hline Sujeto & 1 & 2 & 3 & 4 \\
Edad madre & 27,5 & 39,2 & 38,4 & 39 \\
Riesgo combinado T21 & $>1: 50$ & $1: 62$ & $1: 118$ & $1: 318$ \\
Riesgo del test doble & & & $>1: 50$ & $>1: 50$ \\
Riesgo por edad & $1: 837$ & $1: 101$ & $1: 123$ & $1: 99$ \\
\hline
\end{tabular}

Gráfico 1: Sujetos de riesgo en tamizaje combinado para T21 y riesgo del test doble.

Fuente: Perinatología HSJD

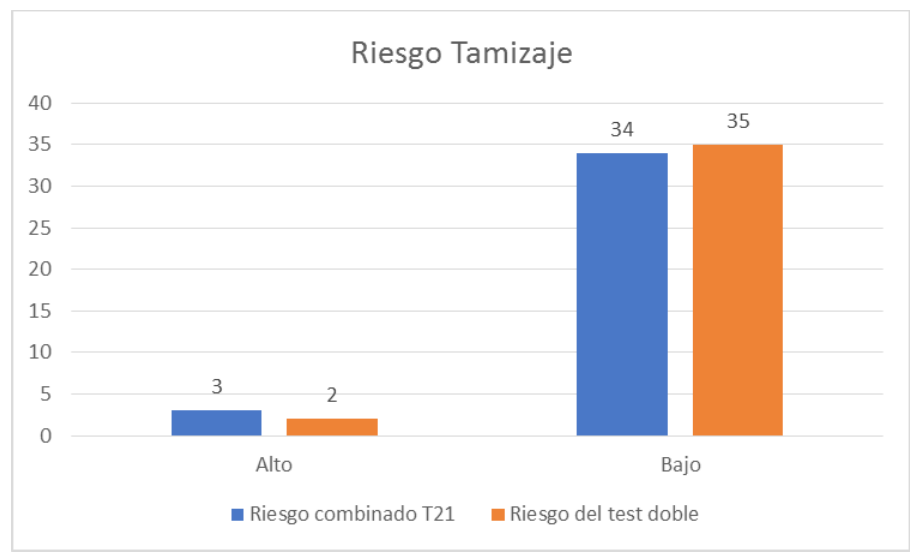


Gráfico 2: Variabilidad de la edad materna.

Fuente: Perinatología HSJD

Tabla 2: Datos analizados en tamizaje combinado.

\begin{tabular}{|lccccc}
\hline \multicolumn{5}{c}{$\begin{array}{c}\beta \text {-HCG libre } \\
\text { MoM }\end{array}$} & \multicolumn{5}{c}{ PAPP-A MoM CRL mm TN mm TN MoM } \\
Promedio & 0,80 & 1,02 & 62,30 & 1,41 & 0,88 \\
Mínimo & 0,2 & 0,27 & 38 & 0,4 & 0,32 \\
Máximo & 2,51 & 3,54 & 82,84 & 3,9 & 2,27 \\
Rango & $0.2-2.51$ & $0.27-3.54$ & $38-82.84$ & $0.4-3.9$ & $0.32-2.27$ \\
\hline
\end{tabular}

Fuente: Perinatología HSJD

Gráfico 3: Hueso nasal.

Fuente: Perinatología HSJD 


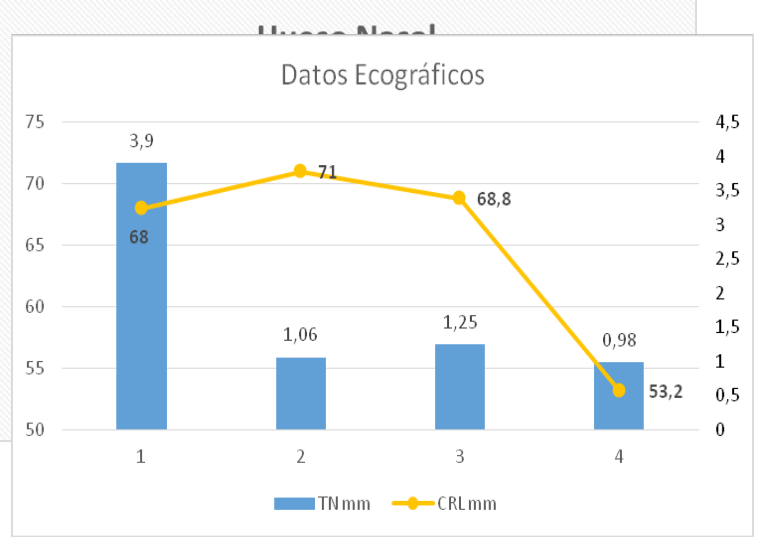

Gráfico 4: Resultados de $\beta$ HCG y PAPP-A compara-

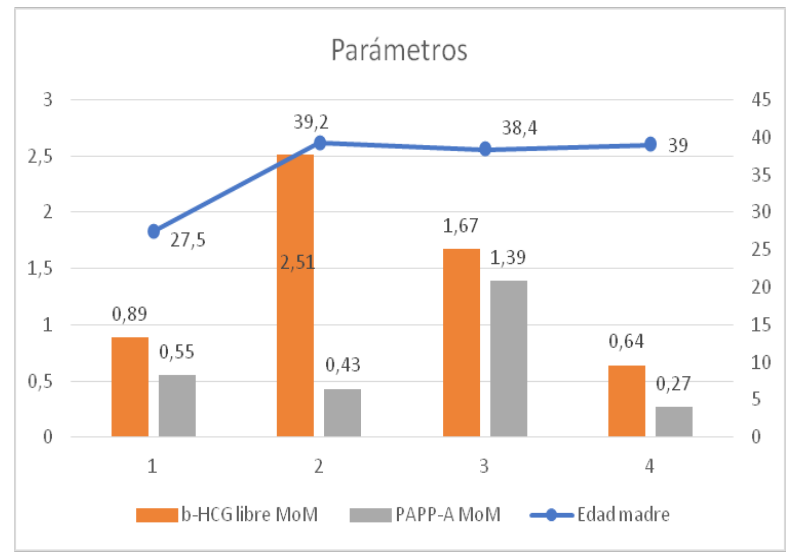

dos con edad materna en sujetos de riesgo.

Fuente: Perinatología HSJD
Gráfico 5: Translucencia nucal y longitud cráneo rabadilla en sujetos de riesgo.

Fuente: Perinatología HSJD 
Tabla 3: Datos analizados por laboratorio.

\begin{tabular}{|c|c|c|c|c|c|}
\hline \multirow[b]{2}{*}{ Promedio } & \multicolumn{2}{|c|}{ Estriol PAPP-A } & $\begin{array}{c}\text { B-HCG } \\
\text { libre } \\
\mathrm{ng} / \mathrm{mL}\end{array}$ & \multicolumn{2}{|c|}{ Alfa Feto Proteína UI/ml HCG mUI/ml } \\
\hline & 0,13 & 5,61 & 28,78 & 15,89 & 87714,7 \\
\hline Mínimo & 0,07 & 1,22 & 7 & 6,36 & 18394 \\
\hline Máximo & 0,6 & 10 & 90 & 33,3 & 78248 \\
\hline
\end{tabular}


Tabla||V: Sujetos de riesgo en anâlisis de tamizaje combinado.

\begin{tabular}{|lcccc|}
\hline Sujeto & 1 & 2 & 3 & 4 \\
Edad madre & 27,5 & 39,2 & 38,4 & 39 \\
Riesgo combinado T21 & $>1: 50$ & $1: 62$ & $1: 118$ & $1: 318$ \\
Riesgo del test doble & & & $>1: 50$ & $>1: 50$ \\
Riesgo por edad & $1: 837$ & $1: 101$ & $1: 123$ & $1: 99$ \\
\hline \hline
\end{tabular}

Fuente: Perinatologia HSJD.

Tabla V: Datos analizados en tamizaje combinado

\begin{tabular}{|lccccc|}
\hline & $\begin{array}{c}\text { p-HCG libre } \\
\text { MoM }\end{array}$ & PAPP-A MoM & CRL mm & TN mm & TN MoM \\
Promedio & 0,80 & 1,02 & 62,30 & 1,41 & 0,88 \\
Mínimo & 0,2 & 0,27 & 38 & 0,4 & 0,32 \\
Máximo & 2,51 & 3,54 & 82,84 & 3,9 & 2,27 \\
Rango & $0.2-2.51$ & $0.27-3.54$ & $38-82.84$ & $0.4-3.9$ & $0.32-2.27$ \\
\hline
\end{tabular}

Fuente: Perinatologia HSJD

Tabla VI: Datos analizados por laboratorio.

\begin{tabular}{|lccccc|}
\hline & $\begin{array}{c}\text { Estriol } \\
\mathbf{n g} / \mathbf{m l}\end{array}$ & $\begin{array}{c}\text { PAPP-A } \\
\text { UI/ml }\end{array}$ & $\begin{array}{r}\text { B-HCG } \\
\text { libre } \mathbf{n g} / \mathbf{m L}\end{array}$ & Alfa Feto Proteína UI/ml & HCG mUI/ml \\
Promedio & 0,13 & 5,61 & 28,78 & 15,89 & 87714,7 \\
Mínimo & 0,07 & 1,22 & 7 & 6,36 & 18394 \\
Máximo & 0,6 & 10 & 90 & 33,3 & 78248 \\
\hline
\end{tabular}

Fuente: Perinatologia HSJD. 
